\title{
Fuzzy Rule Based Systems for Gender Classification from Blog Data
}

\author{
Han Liu ${ }^{1}$ and Mihaela Cocea ${ }^{2}$ \\ ${ }^{1}$ School of Computer Science and Informatics, Cardiff University \\ Queens' Buildings, 5 The Parade, Cardiff, United Kingdom CF24 3AA \\ ${ }^{2}$ School of Computing, University of Portsmouth \\ Buckingham Building, Lion Terrace, Portsmouth, United Kingdom PO1 3HE \\ Email: liuh48@cardiff.ac.uk, mihaela.cocea@port.ac.uk
}

\begin{abstract}
Gender classification is a popular machine learning task, which has been involved in various application areas, such as business intelligence, access control and cyber security. In the context of information granulation, gender related information can be divided into three types, namely, biological information, vision based information and social network based information. In traditional machine learning, gender identification has been typically treated as a discriminative classification task, i.e. it is aimed at learning a classifier that discriminates between male and female. In this paper, we argue that it is not always appropriate to identify gender in the way of discriminative classification, especially when considering the case that both male and female people are of high diversity and thus individuals of different genders could have high similarity to each other in terms of their characteristics. In order to address the above issue, we propose the use of a fuzzy approach for generative classification of gender. In particular, we focus on gender classification based on social network information. We conduct an experiment study by using a blog data set, and compare the fuzzy approach with C4.5, Naive Bayes and Support Vector Machine in terms of classification performance. The results show that the fuzzy approach outperforms the other approaches and is also capable of capturing the diversity of both male and female people and dealing with the fuzziness in terms of gender identification.
\end{abstract}

Keywords-Data Mining; Machine Learning; Fuzzy Rule Based Systems; Text Classification; Gender Classification

\section{INTRODUCTION}

Gender classification is aimed at identifying the gender of a person, i.e. it is to determine person is male or female. In practice, gender classification can sever various applications, such as business intelligence [1], access control [2] and security checks [3].

Gender classification can be done through manual classification by using expert knowledge or automatic classification by learning classifiers from real data. Due to the vast and rapid increase in the size of data, machine learning has become an increasingly more popular tool for gender identification. Some popular learning approaches reported in [4] include support vector machine (SVM) [5], k nearest neighbour (KNN) [6] and Gaussian mixture models (GMM) [7] .

From granular computing perspectives, gender related information can be decomposed into biological information (e.g. EEG and DNA), vision based information (e.g. height and hair length) and social network information (e.g. Facebook posts, tweets and blogs). From this point of view, gender classification can be achieved by learning classifiers from data obtained from different sources, such as biological data, images and text, i.e. different types of features are extracted for training gender classifiers.

In traditional machine learning, gender identification has been typically treated as a discriminative classification task, due to the case that the two classes (male and female) are considered to be mutually exclusive. However, in reality, both male and female people are of high diversity and can be divided into many different groups, which indicates that individuals of different genders may have high similarity to each other in terms of their characteristics. It is also possible that a person of one gender intentionally shows characteristics of the other gender, e.g. they may try to disguise themselves.

On the basis of the above argumentation, it is not always appropriate to treat gender identification as a discriminative classification task. Instead, generative classification is considered to be more suitable for such classification tasks. In this paper, we propose the use of fuzzy approaches for generative classification, and focus the study on gender identification based on features extracted from online text.

The rest of this paper is organized as follows: Section II provides related work on gender classification, feature extraction from text and fuzzy classification. In section III, we present a fuzzy approach in terms of its key features, and justify why fuzzy approaches would be more suitable for gender identification from textual data. In Section IV, we report an experimental study conducted by using a blog gender data set and discuss the results to show the advantages of the fuzzy approach. In Section V, we summarize the contributions of this paper and suggest further directions towards advancing this research area in the future.

\section{RELATED WORK}

In this section, we provide an overview of gender classification in the context of text mining and review popular methods of feature extraction in the area of text classification. Also, we provide the background and recent developments of fuzzy text classification. 


\section{A. Review of Feature Extraction Methods}

Feature extraction from textual data consists of four stages: enrichment, pre-processing, transformation and vectoring [8].

The enrichment stage aims at assigning semantic information through recognition and tagging of named entities (NE) in order to support term filtering in the later stages. Popular taggers include Part of Speech (POS) Tagger, Abner Tagger and Dictionary Tagger. More details on text enrichment can be found in [8].

Pre-processing aims to filter those irrelevant terms such as stop words, punctuation, numbers and $\mathrm{N}$-Char words (each word that contains no more than $\mathrm{n}$ characters) [8]. Also, it is necessary to covert upper cases to lower cases and remove endings through word stemming [8].

Transformation aims to transform textual data into structured data in order to enable the direct use of machine learning algorithms for sentiment classification. In particular, the bagof-words (BOW) method is one of the most popular ways to achieve such transformation [9], [10] by turning each single word in a document (textual instance) into a single attribute (feature) in the transformed data set.

In the above context, each word, which is used as a feature, is viewed as a single-word term. However, a term can also consists of multiple words (i.e. multi-word term), when NGram (an extension of BOW) is used for transforming textual data into structured data.

Following the above transformation, it is also necessary to count the frequency of each term in order to enable feature selection by filtering those less frequently occurring terms. In this way, the data dimensionality can be reduced significantly leading to more efficient processing in the later stages.

In the vectoring stage, each term is turned into either a binary or numerical feature. For a binary feature, the Boolean value indicates the presence or absence of the corresponding term in a specific document. For a numerical feature, the frequency of the corresponding term is used as the value of the feature in the learning stage.

For BOW, there are four types of frequency, namely, term absolute frequency, term relevant frequency, inverse document frequency and inverse class frequency. For N-Gram, there are three types of frequency, namely, corpus frequency, document frequency and sentence frequency. More details on these types of frequency can be found in [8].

\section{B. Overview of Gender Classification}

In the context of text mining, gender classification is typically achieved by learning classifiers from text posted on social networks, such as emails, Facebook posts, tweets and blogs.

In [4], Lin et al listed several representative studies on gender classification through using daily information posted via social network platforms, and reported that the classification accuracy was relatively low, in comparison with using features extracted from biological data and images.

In particular, an investigation was conducted in [11] for mining gender attribution of authorship from emails. In this investigation, SVM was used to learn classifiers from manually extracted features of content-free emails, e.g. style markers, structural characteristics, and gender-preferential language features, and the classification accuracy was about 70\% [4].

Another study was conducted in [12] by using a real-life blog data set. In this study, an ensemble feature selection approach was proposed, and SVM and Naive Bayes (NB) were used together for learning classifiers, which led to the classification accuracy of $88.56 \%$.

Overall, gender classification through using social network based information is generally more difficult than using other sources of information. As reported in [4], the number of features extracted from social network data is very high and the number of instances is also massive, which could lead to high computational complexity and affect the learning performance due to the presence of more irrelevant features. Also, by its nature, text is characterized by fuzziness, imprecision and uncertainty, which leads to further difficulty in identifying gender from social network based information.

\section{Background of Fuzzy Text Classification}

In the area of text classification, a review of fuzzy approaches for natural language processing (NLP) was made in [13] in 2012, which highlighted that there was a very low percentage of papers relating to fuzzy approaches over all the papers published in the NLP area and that there were very few NLP related application papers published in the area of fuzzy systems. Following the publication of the above review paper, a number of fuzzy approaches have been proposed for various applications, since fuzzy approaches are more suitable to deal with the ambiguity and fuzziness of text.

A fuzzy approach was developed in [14] for classification of companies based on fuzzy fingerprint text. The classification results showed that the fuzzy approach outperformed the commonly used non-fuzzy approaches. Another fuzzy approach was used in [15] for automatically building a corpus for comparison of text similarity. The results reported in [15] showed that the fuzzy metrics had a higher correlation with human ratings in comparison with the traditional metrics. An unsupervised fuzzy approach was used in [16] for classification of Twitter users according to their gender.

On the other hand, a fuzzy rule based approach was proposed in [17] for addressing the model complexity issue, and the experimental results showed that the fuzzy approach led to a reduction in computational complexity, while maintaining a similar classification performance, when comparing with other non-fuzzy approaches popularly used for text classification. Based on this work, the fuzzy approach was investigated further in [18] for discussing how the membership degree values can be used for more refined outputs, which could reflect different intensities of sentiment.

\section{Fuzzy Rule Based Classification}

In this section, we provide theoretical preliminaries relating to fuzzy logic and illustrate the procedure of using a fuzzy rule based system for classification tasks. Also, we justify why fuzzy approaches are more suitable for gender classification than those popularly used non-fuzzy approaches. 


\section{A. Theoretical Preliminaries}

Fuzzy logic is an extension of deterministic logic. In this context, a truth value is ranged from 0 to 1 rather binary ( 0 or 1). Fuzzy logic theory is mainly used in the contexts of fuzzy sets and fuzzy rule based systems.

In the context of fuzzy sets, each element $e_{i}$ in a set $S$ has a certain degree of membership $f_{S}\left(e_{i}\right)$, where $f_{S}\left(e_{i}\right) \in[0,1]$ and $1 \leq i \leq n$. In other words, a fuzzy set employs a soft boundary determining the membership or non-membership of each element to the set.

In the context of fuzzy rule based systems, the main operation is to transform each continuous attribute into a number $(n)$ of linguistic attributes towards learning of a set of fuzzy rules. In particular, each linguistic attribute transformed from a continuous attribute is essentially a fuzzy set defined with a membership function that maps the crisp value of the continuous attribute into a membership degree value (the value of the linguistic attribute).

Membership functions could be of different shapes, such as trapezoidal, triangular and rectangular membership functions. In general, a trapezoidal membership function can be viewed as a generalization of triangular and rectangular membership functions. In fact, defining a membership function is essentially aimed at estimating four parameters (a, b, c, d), as illustrated below and in Fig. 1.

$$
f_{T}(x)= \begin{cases}0, & \text { when } x \leq a \text { or } x \geq d \\ (x-a) /(b-a), & \text { when } a<x<b \\ 1, & \text { when } b \leq x \leq c \\ (d-x) /(d-c), & \text { when } c<x<d\end{cases}
$$

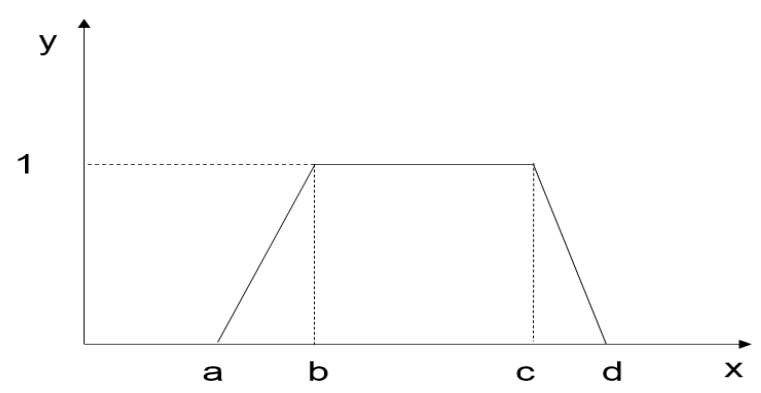

Fig. 1. Trapezoid Fuzzy Membership Function [17]

As shown in Fig 1, if $b=c$, then the shape of the membership function would be triangular. Similarly, if $a=b$ and $c=d$, then the shape of the membership function would be rectangular.

In practice, the parameters of a membership function can be estimated by using expert knowledge [19] or through learning statistically from data [20], [21].

\section{B. Procedure}

In the classification stage, a fuzzy rule based system involves five main operations: fuzzification, application, implication, aggregration and defuzzification. We illustrate the whole procedure by using the following example of fuzzy rules:
- Rule 1: if $x_{1}$ is Young and $x_{2}$ is Cold then class=Yes;

- Rule 2: if $x_{1}$ is Young and $x_{2}$ is Warm then class=Yes;

- Rule 3: if $x_{1}$ is Young and $x_{2}$ is Hot then class=No;

- Rule 4: if $x_{1}$ is Middle-aged and $x_{2}$ is Cold then class $=\mathrm{No}$;

- Rule 5: if $x_{1}$ is Middle-aged and $x_{2}$ is Warm then class=Yes;

- Rule 6: if $x_{1}$ is Middle-aged and $x_{2}$ is Hot then class=No;

- Rule 7: if $x_{1}$ is Old and $x_{2}$ is Cold then class=No;

- Rule 8: if $x_{1}$ is Old and $x_{2}$ is Warm then class=No;

- Rule 9: if $x_{1}$ is Old and $x_{2}$ is Hot then class=No;

The fuzzy membership functions defined for the linguistic attributes transformed from $x_{1}$ and $x_{2}$ are illustrated in Fig. 2 and Fig. 3, respectively.

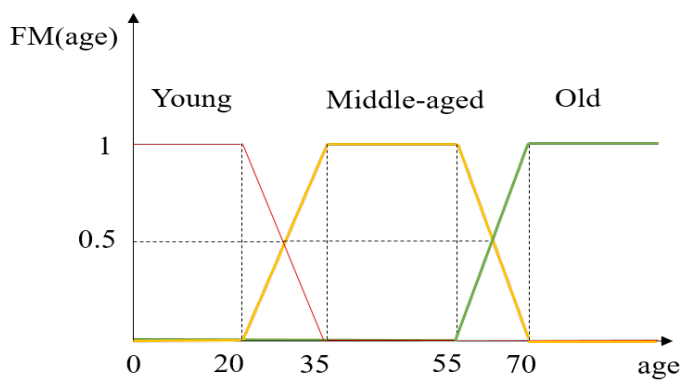

Fig. 2. Fuzzy membership functions for age

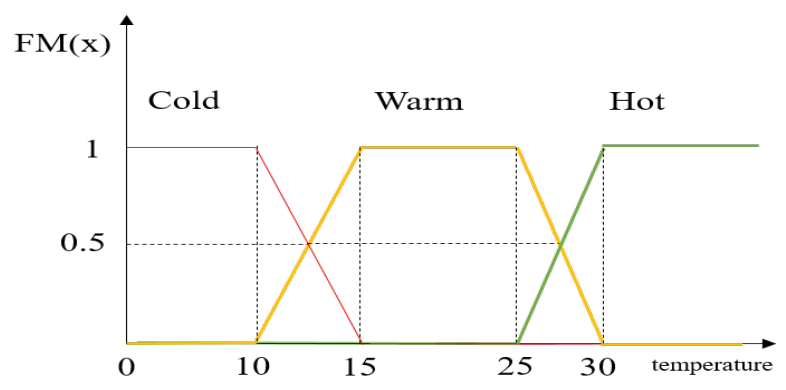

Fig. 3. Fuzzy membership functions for temperature

According to Fig. 2 and Fig. 3, if $x_{1}=30$ and $x_{2}=28$, then the following steps will be executed:

Fuzzification:

Rule 1: $f_{\text {Young }}(30)=0.67, f_{\text {Cold }}(28)=0$;

Rule 2: $f_{\text {Young }}(30)=0.67, f_{\text {Warm }}(28)=0.4$;

Rule 3: $f_{\text {Young }}(30)=0.67, f_{\text {Hot }}(28)=0.6$;

Rule 4: $f_{\text {Middle-aged }}(30)=0.33, f_{\text {Cold }}(28)=0$;

Rule 5: $f_{\text {Middle-aged }}(30)=0.33, f_{W \text { arm }}(28)=0.4$;

Rule 6: $f_{\text {Middle-aged }}(30)=0.33, f_{\text {Hot }}(28)=0.6$;

Rule 7: $f_{\text {Old }}(30)=0, f_{\text {Cold }}(28)=0$;

Rule 8: $f_{\text {Old }}(30)=0, f_{\text {Warm }}(28)=0.4$;

Rule 9: $f_{\text {Old }}(30)=0, f_{\text {Hot }}(28)=0.6$;

In the fuzzification step, the notation $f_{\text {Warm }}(28)=0.4$ represents that the membership degree of the numerical value 
' 28 ' to the fuzzy set defined with the linguistic attribute 'Warm' is 0.4. The fuzzification step is aimed at mapping the value of a continuous attribute to a value of membership degree to a fuzzy set (i.e. mapping to the value of a linguistic attribute transformed from the continuous attribute).

Application:

Rule 1: $f_{\text {Young }}(30) \wedge f_{\text {Cold }}(28)=\operatorname{Min}(0.67,0)=0$;

Rule 2: $f_{\text {Young }}(30) \wedge f_{\text {Warm }}(28)=\operatorname{Min}(0.67,0.4)=0.4$;

Rule 3: $f_{\text {Young }}(30) \wedge f_{\text {Hot }}(28)=\operatorname{Min}(0.67,0.6)=0.6$;

Rule 4: $f_{\text {Middle-aged }}(30) \wedge f_{\text {Cold }}(28)=\operatorname{Min}(0.33,0)=0$;

Rule 5: $f_{\text {Middle-aged }}(30) \wedge f_{W a r m}(28)=\operatorname{Min}(0.33,0.4)=$ 0.33 ;

Rule 6: $f_{\text {Middle-aged }}(30) \wedge f_{H o t}(28)=\operatorname{Min}(0.33,0.6)=$ 0.33 ;

Rule 7: $f_{\text {Old }}(30) \wedge f_{\text {Cold }}(28)=\operatorname{Min}(0,0)=0$;

Rule 8: $f_{\text {Old }}(30) \wedge f_{W a r m}(28)=\operatorname{Min}(0,0.4)=0$;

Rule 9: $f_{\text {Old }}(30) \wedge f_{H o t}(28)=\operatorname{Min}(0,0.6)=0$;

In the application step, the conjunction of the two fuzzy membership degrees respectively for the two attributes ' $x_{1}$ and ' $x_{2}$ ' is aimed at deriving the firing strength of a fuzzy rule. For example, the antecedent of Rule 3 consists of $x_{1}$ is Young and $x_{2}$ is Hot, so the firing strength of Rule 3 is 0.6, while $f_{\text {Young }}(30)=0.67$ and $f_{\text {Hot }}(28)=0.6$.

Implication:

Rule 1: $f_{\text {Rule } 1 \rightarrow Y \text { es }}(30,28)=0$;

Rule 2: $f_{\text {Rule } 2 \rightarrow \text { Yes }}(30,28)=0.4$;

Rule 3: $f_{\text {Rule } 3 \rightarrow N o}(30,28)=0.6$;

Rule 4: $f_{\text {Rule } 4 \rightarrow \text { No }}(30,28)=0$;

Rule 5: $f_{\text {Rule } 5 \rightarrow \text { Yes }}(30,28)=0.33$;

Rule 6: $f_{\text {Rule } 6 \rightarrow \text { No }}(30,28)=0.33$;

Rule 7: $f_{\text {Rule } \rightarrow \text { No }}(30,28)=0$;

Rule 8: $f_{\text {Rule } 8 \rightarrow N o}(30,28)=0$;

Rule 9: $f_{\text {Rule } 9 \rightarrow N o}(30,28)=0$;

In the implication step, the firing strength of a fuzzy rule derived in the application step can be used further to identify the membership degree of the value of an input vector to the class label 'Yes' or 'No', depending on the consequent of the fuzzy rule. For example, $f_{\text {Rule } 2 \rightarrow Y e s}(30,28)=0.4$ indicates that the consequent of Rule 2 is assigned the class label 'Yes' and the input vector ' $(30,28)$ ' has the membership degree of 0.4 to the class label 'Yes'. In other words, the inference through Rule 2 leads to the input vector ' $(30,28)$ ' having the membership degree value of 0.4 to the class label 'Yes'.

Aggregation:

$f_{Y e s}(30,28)=f_{\text {Rule } 1 \rightarrow \text { Yes }}(30,28) \vee f_{\text {Rule } 2 \rightarrow \text { Yes }}(30,28) \vee$ $f_{\text {Rule } 5 \rightarrow \text { Yes }}(30,28)=\operatorname{Max}(0,0.4,0.33)=0.4$

$f_{\text {No }}(30,28)=f_{\text {Rule } 3 \rightarrow \text { No }}(30,28) \vee f_{\text {Rule } 4 \rightarrow \text { No }}(30,28) \vee$ $f_{\text {Rule } 6 \rightarrow \text { No }}(30,28) \quad \vee \quad f_{\text {Rule } 7 \rightarrow \text { No }}(30,28)$

$f_{\text {Rule } 8 \rightarrow \text { No }}(30,28) \vee f_{\text {Rule } 9 \rightarrow \text { No }}(30,28)$

$=\operatorname{Max}(0.6,0,0.33,0,0,0)=0.6$
In the aggregation step, the membership degree value of the input vector to the class label ('Yes' or 'No'), which is inferred through a rule, is compared with the other membership degree values inferred through the other rules, towards finding the maximum among all the membership degree values. For example, Rule 1, Rule 2 and Rule 5 are all assigned the class label 'Yes' as their consequent and the membership degree values of the input vector ' $(30,28)$ ' inferred through the three rules are $0,0.4$ and 0.33 , respectively, to the class label 'Yes'. As the maximum of the fuzzy membership degree values is 0.4 , the input vector is considered to have the membership degree value of 0.4 to the class label 'Yes'.

Defuzzification:

$f_{\text {No }}(30,28)>f_{\text {Yes }}(30,28) \rightarrow$ class $=N o$;

In the defuzzification step, the aim is to identify the class label to which the input vector has the highest value of membership degree. In this example, as the membership degree value of the input vector $(30,28)$ to the class label 'No' is 0.6 , which is higher than the membership degree (0.4) to the class label 'Yes', the final output is 'No' towards classifying the unseen instance $(30,28, ?)$.

\section{Justification}

We propose the use of fuzzy rule based systems for gender classification based on social network information, due to the advantages of fuzzy logic and its suitability for text processing, as outlined below.

Firstly, fuzzy logic is well capable of dealing with the fuzziness, imprecision and uncertainty of text. In particular, it considers classification to be a 'degree of grey' problem rather than a 'black and white' problem (currently used in text classification). This way of defining the classification problem leads to a reduction of bias on both male and female classes. For example, popular algorithms for text classification, such as C4.5, NB and SVM, deal with continuous attributes by setting up crisp intervals, each of which is used to judge whether a condition is met through checking the values of the continuous attributes, towards classifying unseen instances. The above way of dealing with continuous attributes has been generally criticized as judgment bias in fuzzy systems literature, which can be replaced with using fuzzy intervals.

Secondly, fuzzy approaches work in the way of generative learning rather than discriminative learning (typically used for training gender classifiers). In other words, fuzzy approaches aim at learning classifiers that consider each class equally, through measuring the membership degree value of an instance to each class independently, whereas those popularly used nonfuzzy approaches aim at learning classifiers that discriminate one class from the the other class, towards uniquely classifying an instance. In the context of gender classification, male and female people could have some shared language terms in writing blogs and posts [4]. Also, people of different genders may learn from each other in terms of writing style. Furthermore, it 
is possible in reality that people may try to disguise themselves by showing intentionally the characteristics of the other gender in terms of writing style.

Thirdly, both male and female people are of high diversity in the world, i.e. people of each gender can be divided into different groups. From granular computing perspectives, each group of people can be viewed as a subclass of the male or female class. In real applications, it is unlikely that a training set can represent the full population of male and female people. From this point of view, each class (male or female) assigned to a training instance would actually represent a subclass of the male or female class, so an unseen instance may not belong to either one of the two classes, due to the case that the instance belongs to another subclass that is not included in the training set. When the above case arises, fuzzy approaches are capable of capturing it through showing that the instance has the membership degree value of 0 to both classes [18]. In contrast, discriminative approaches cannot capture the above case, due to their nature of training classifiers to discriminate between the two classes.

\section{EXPERIMENTAL SETUP AND RESUlts}

In this section, we report an experimental study conducted by using a blog gender data set [12]. The data set contains 3226 blogs (1551 male and 1672 female).

In terms of classification performance, we compare the fuzzy approach with SVM, NB and C4.5, while three different types of features are extracted, namely, uni-gram (1-word term), bi-gram (2-word term) and tri-gram (3-word term). The results are shown in Table I.

TABLE I

Classification ACCURACy

\begin{tabular}{c|c|c|c|c}
\hline Feature Extraction & C4.5 & NB & SVM & Fuzzy \\
\hline Uni-gram & 0.559 & 0.662 & 0.508 & 0.776 \\
Bi-Gram & 0.535 & 0.579 & 0.573 & 0.892 \\
Tri-gram & 0.641 & 0.692 & 0.781 & 0.806 \\
\hline
\end{tabular}

Table I shows that the fuzzy approach outperforms significantly the other three non-fuzzy approaches in all the cases. The results are likely due to the case that a fuzzy classifier is not biased on one of the two classes but judges independently the degree to which an instance belongs to each class. As argued in Section III-C, individuals of different genders may have high similarity to each other in terms of writing style, which indicates that the two classes (male and female) would have overlaps. In this case, the nature of generative learning through fuzzy approaches makes it achievable to capture that highly similar patterns (writing styles) exist in blogs posted by both male and female authors.

In terms of feature extraction, the results show that the extraction of Bi-grams (2-word terms) leads to the best performance of the fuzzy approach, which are likely due to the case that the extraction of 2 -word terms results in more features of higher frequency, leading to more useful and confident information (reflecting gender characteristics) being captured. In contrast, the use of 1-word terms could lead to loss of some important information, since multi-word terms are generally more informative than single-word terms. In addition, increasing the number of combined words (for making up a term) generally results in the decrease of term frequency, leading to the extracted features being less useful.

TABLE II

MEMBERSHIP DEGREE

\begin{tabular}{c|c|c|c|c}
\hline No & Class & FM(Class=M) & FM(Class=F) & Prediction(Class) \\
\hline 1 & M & 1 & 0 & M \\
2 & F & 0 & 1 & F \\
3 & F & 0.33 & 0.67 & F \\
4 & F & 0.5 & 0.5 & F \\
5 & M & 0.67 & 0 & M \\
6 & M & 0.17 & 0 & M \\
7 & F & 0 & 0.5 & F \\
8 & M & 1 & 0.43 & M \\
9 & M & 1 & 1 & F \\
10 & F & 1 & 1 & $?$ \\
11 & M & 0 & 0 & $?$ \\
12 & F & 0 & 0 &
\end{tabular}

The membership degree values of instances (selected as representative examples) to the two classes are shown in Table II. The results show diverse cases of gender classification. In particular, the first two cases (row 1 and row 2) indicate that the fuzzy classifier judges that the instance fully belongs to the male or female class, i.e. only characteristics of one gender are captured by the classifier and these characteristics are uniquely originated from people of one gender. The third case indicates that the fuzzy classifier captures both male and female characteristics from a blog posted by a female person, but the majority of the characteristics match the ones of female. The fourth case indicate that the fuzzy classifier captures characteristics that $50 \%$ match both male and female.

The above cases show that the sum of the membership degree values of an instance to two classes is 1 . However, the sum is not necessarily equal to 1 , i.e. it could be greater or less than 1. In particular, the fifth and sixth cases indicate that the fuzzy classifier captures characteristics of male only but the characteristics do not fully match the ones of male, i.e. for the fifth case the degree of matching is higher, but for the sixth case the degree is much lower. Also, the seventh case indicates that the fuzzy classifier capture characteristics of female only with the matching degree of 0.5 . The above phenomenon can be explained by the commonsense that people of the same gender present different intensities of the characteristics originated from the majority of people of this gender.

The eighth case indicates that the fuzzy classifier captures characteristics that fully match the ones of male but also partially match the ones of female. This could be partially explained by the point (mentioned in Section III-C) that people of different genders have shared language styles in writing blogs. From this point of view, the author of the blog strongly presents the characteristics of male styled writing but the writing style also has some similarity to the one of female. The 9 th and 10th cases indicate that the fuzzy classifier captures characteristics that fully match the ones of both male and female. The above phenomenon could be explained by two 
points: a) a blog is written fully in shared language terms; b) a person of one gender presents in full the characteristics of the other gender in terms of writing style, which results in discovery of highly similar or even the same pattern from blogs posted by both male and female people.

The last two cases indicate that the fuzzy classifier judges that the instances do not belong to either one of the two classes, i.e. none of the gender characteristics, which are discovered from the training instances (blogs), are captured from the unseen instances. This is likely due to the high diversity of people. As mentioned in Section III-C, both male and female people can be subdivided into different groups, which are viewed as sub-classes of the male or female class. In real applications, it is likely that the training data only represents one or more (not all) groups of male and female people, which leads to the situation that an unseen instance belongs to another sub-class of the male or female class but the sub-class is absent from the training set.

\section{CONCLUSiON}

In this paper, we proposed the use of fuzzy approaches for gender classification. In particular, we treat gender identification as a task of generative classification instead of discriminative classification. We compared the fuzzy approach with popularly used discriminative approaches (SVM, NB and C4.5), in terms of classification accuracy. The results show that the fuzzy approach outperforms the other three ones.

We also reported the results on fuzzy membership degree values of instances to two classes (male and female). The results show diverse cases of gender classification. In particular, individuals of different genders could have high similarity to each other in terms of their writing style. Also, due to the high diversity of people, it is likely that the training data does not represent a full population of male and female people, which could result in the case that a person does not present any characteristics that match the ones of male or female discovered from the training instances. Furthermore, it is also possible that the writing style captured from a blog matches fully the characteristics of shared language terms rather than any characteristics of a specific gender. In addition, it is also possible in reality that a person of one gender tries to disguise themselves by presenting the characteristics of the other gender. All of the above cases can be captured by using fuzzy approaches through identifying the membership degree values of an instance to the male and female classes.

In future, we will investigate how to achieve effective gender identification through using granular computing concepts. For example, due to the high diversity of people, both the male and female classes can be specialized/decomposed into subclasses through information granulation [22]. Since the classes and sub-classes are located in different levels of granularity, traditional gender classification tasks can thus be extended in the setting of multi-granularity learning.

\section{ACKNOWLEDGMENT}

The authors acknowledge support for the research reported in this paper through the Research Development Fund at the University of Portsmouth.

\section{REFERENCES}

[1] G. Guo, "Human age estimation and sex classification," in Video Analytics for Business Intelligence, vol. 409, 2012, pp. 101-131.

[2] — - "Gender classification," in Encyclopedia of Biometrics, 2014, pp $1-6$.

[3] N. Ali and L. Xavier, "Person identification and gender classification using gabor filters and fuzzy logic," International Journal of Electrical, Electronics and Data Communication, vol. 2, no. 4, pp. 20-23, April 2014.

[4] F. Lin, Y. Wu, Y. Zhuang, X. Long, and W. Xu, "Human gender classification: a review," International Journal of Biometrics, vol. 8, no. 3-4, 2016

[5] J. A. Suykens and J. Vandewalle, "Least squares support vector machine classifiers," Neural processing letters, vol. 9, no. 3, pp. 293-300, 1999.

[6] L. E. Peterson, "K-nearest neighbor," Scholarpedia, vol. 4, no. 2, p. 1883, 2009.

[7] S. Mitra and M. Savvides, "Gaussian mixture models based on the frequency spectra for human identification and illumination classification," in IEEE Workshop on Automatic Identification Advanced Technologies, 2005, pp. 245-250.

[8] K. Thiel and M. Berthold, "The knime text processing feature: An introduction," KNIME, Tech. Rep., 2012.

[9] K. Reynolds, A. Kontostathis, and L. Edwards, "Using machine learning to detect cyberbullying," in International Conference on Machine Learning and Applications, December 2011, pp. 241-244.

[10] R. Zhao, A. Zhou, and K. Mao, "Automatic detection of cyberbullying on social networks based on bullying features," in International Conference on Distributed Computing and Networking, 2016.

[11] M. Corney, O. de Vel, A. Anderson, and G. Mohay, "Gender-preferential text mining of e-mail discourse," in Computer Security Applications Conference, 2002, pp. 282-289.

[12] A. Mukherjee and B. Liu, "Improving gender classification of blog authors," in Conference on Empirical Methods in natural Language Processing, 2010, pp. 207-217.

[13] J. P. Carvalho, F. Batista, and L. Coheur, "A critical survey on the use of fuzzy sets in speech and natural language processing," in IEEE International Conference on Fuzzy Systems, Brisbane, QLD, Australia, 10-15 June 2012.

[14] F. Batista and J. P. Carvalho, "Text based classification of companies in crunchbase," in IEEE International Conference on Fuzzy Systems, Istanbul, Turkey, 2-5 August 2015.

[15] D. Chandran, K. A. Crockett, D. Mclean, and A. Crispin, "An automatic corpus based method for a building multiple fuzzy word dataset," in IEEE International Conference on Fuzzy Systems, Istanbul, Turkey, 2-5 August 2015.

[16] M. Vicente, F. Batista, and J. P. Carvalho, "Twitter gender classification using user unstructured information," in IEEE International Conference on Fuzzy Systems, Istanbul, Turkey, 2-5 August 2015.

[17] H. Liu and M. Cocea, "Fuzzy rule based systems for interpretable sentiment analysis," in International Conference on Advanced Computational Intelligence, Doha, Qatar, 4-6 February 2017, pp. 129-136.

[18] C. Jefferson, H. Liu, and M. Cocea, "Fuzzy approach for sentiment analysis," in IEEE International Conference on Fuzzy Systems, Naples, Italy, 9-12 July 2017.

[19] E. Mamdani and S. Assilian, "An experiment in linguistic synthesis with a fuzzy logic controller," International Journal of Human-Computer Studies, vol. 51, no. 2, pp. 135-147, 1999.

[20] S.-M. Chen, "A fuzzy reasoning approach for rule-based systems based on fuzzy logics," IEEE Transactions on Systems, Man and Cybernetics - Part B: Cybernetics, vol. 26, no. 5, pp. 769-778, October 1996.

[21] F. Bergadano and V. Cutello, "Learning membership functions," in European Conference on Symbolic and Quantitative Approaches to Reasoning and Uncertainty, Granada, Spain, 8-10 November 1993, pp. 25-32.

[22] H. Liu and M. Cocea, "Semi-random partitioning of data into training and test sets in granular computing context," Granular Computing, vol. 2, no. 4, pp. 357-386, 2017. 